\title{
Efficient Full-wave Modal Analysis of Arbitrarily Shaped Waveguides using BI-RME and Nyström Methods
}

\author{
Máriam Taroncher ${ }^{1}$, Ana Vidal ${ }^{1}$, Vicente E. Boria ${ }^{1}$, Stephan Marini ${ }^{1}$, Pablo Soto ${ }^{1}$, \\ Santiago Cogollos ${ }^{1}$, Benito Gimeno ${ }^{2}$ \\ ${ }^{1}$ Departamento de Comunicaciones, Univ. Politécnica de Valencia, E-46022 Valencia, Spain, +34 963879718 \\ ${ }^{2}$ Departamento de Física Aplicada y Electromagnetismo, Univ. de Valencia, E-46100 Valencia, Spain.
}

\begin{abstract}
This paper describes a novel technique for the very efficient and accurate full-wave modal analysis of cylindrical waveguides with arbitrary cross-section. This new technique relies on solving the integral equations that provide the well-known Boundary Integral - Resonant Mode Expansion (BI-RME) method by the Nyström approach, instead of using the traditional Galerkin version of the Method of Moments (MoM), thus providing large savings on computational costs. Accuracy aspects of this simple and fast procedure, which are directly connected to the rigorous treatment of the singular behaviour of the integral equation kernels, are carefully considered for waveguides defined by straight, circular and/or elliptical arcs. Comparative benchmarks between the new technique and the original BI-RME method are successfully presented for single- and multi-ridged waveguides, elliptical waveguides and rectangular waveguides with rounded corners.
\end{abstract}

\section{INTRODUCTION}

Arbitrarily shaped waveguides, whose cross-section is defined by linear, circular and/or elliptical arcs, are usually found in many passive waveguide devices used in real applications [1]. For example, ridge rectangular or circular waveguides are frequently present in dual mode filters, and multi-ridged waveguides are the key element of doubly corrugated chokes. Elliptical waveguides have also found increasing application in many passive waveguide devices, such as dual and triple mode filters, circular waveguide polarizers, twists and mode launchers, radiators, resonators and corrugated horns. Finally, the accurate consideration of rounded corners in passive rectangular waveguide devices, usually attributed to present manufacturing techniques, is currently under investigation.

The Computer Aided Design (CAD) tools of these complex passive waveguide devices require a very accurate full-wave modal solution of the above mentioned arbitrary waveguides. Among the many methods proposed in the literature, the BI-RME technique provides the complete modal spectrum of such waveguides [2], as well as the coupling coefficients between these modes and the ones of a surrounding standard rectangular waveguide [3], in a very accurate way. This technique is based on the solution of integral equations by the Galerkin version of MoM, which therefore requires to solve simple and double integrals for computing the matrix elements (see details in [1]).

During the design process of complex waveguide components, the repeated use of the accurate BI-RME approach [2,3] can lead to unaffordable computation times. To alleviate this situation, some recently developed optimization algorithms, e.g. the Aggressive Space Mapping (ASM) technique [4], propose the use of two different simulation tools: an efficient and not very accurate simulation tool, which will support the burden of the design process, and a very accurate but not efficient analysis tool to guarantee the accuracy of the whole process. Therefore, a fast and simple implementation of the BI-RME method is required.

In this paper, a more efficient BI-RME method, based on the Nyström technique implemented with a very simple quadrature rule, is completely described. The accuracy and efficiency improvement provided by this new method is proved through successful comparisons with numerical data obtained with a revisited implementation of the classical BI-RME approach [5].

\section{BASIC THEORY}

In this section, the combination of the BI-RME method with the Nyström technique is described. The novel technique is applied to the solution of the TE and TM modes of arbitrary waveguides, whose contour can be defined by any combination of straight, circular and/or elliptical arcs. Once the modal chart of these waveguides is computed, the coupling integrals between such modes and the ones of a surrounding standard rectangular waveguide can easily be obtained following [3].

The electric field at a generic observation point $\mathbf{r}$ inside the waveguide may be represented as

$$
\mathbf{E}(\mathbf{r})=-\dot{\eta} k \int_{\sigma} \mathbf{G}_{e}\left(\mathbf{r}, \mathbf{s}^{\prime}, k\right) \cdot \mathbf{J}_{\sigma}\left(l^{\prime}\right) d l^{\prime}
$$

where $\sigma$ is the perturbing line that fixes the contour of the arbitrary waveguide and is defined by the parametric equation $\mathbf{s}^{\prime}=\mathbf{s}\left(l^{\prime}\right), \mathbf{G}_{e}$ is the two-dimensional dyadic Green's function of the electric type for the twodimensional resonator and $\mathbf{J}_{\sigma}$ is the current density on $\sigma$.

\section{A. TM Modes}

To compute the TM modes, the BI-RME technique is followed, thus leading to an integral equation after 
imposing the boundary condition to the axial component of the electric field (see (9a) and (9b) in [2]). For solving such equation, instead of using standard Galerkin as in [2], a Nyström quadrature is employed, thus leading to the following algebraic equation

$$
\begin{gathered}
\sum_{\substack{j=1 \\
j \neq i}}^{N} \omega_{i j} g\left(\mathbf{s}_{i}, \mathbf{s}_{j}\right) b_{j}^{\prime}+\omega_{i i} b_{i}^{\prime}+\sum_{m=1}^{M} \frac{\psi_{m}\left(\mathbf{s}_{i}\right)}{k_{m}^{\prime 2}} a_{m}^{\prime}=0 \quad(i=1, . ., N) \\
\left(1-\frac{k^{2}}{k_{m}^{\prime 2}}\right) \cdot a_{m}^{\prime}=k^{2} \sum_{j=1}^{N} \gamma_{j} \frac{\psi_{m}\left(\mathbf{s}_{j}\right)}{k_{m}^{\prime 2}} b_{j}^{\prime} \quad(m=1, \ldots, M)
\end{gathered}
$$

where $\omega_{j i}$ and $\gamma_{j}$ are Nyström quadrature weights, $N$ is the number of points used for the subdivision of the perturbing contour, $\mathbf{s}_{i}$ and $\mathbf{s}_{j}$ are the discrete observation and source points, $g$ is the rapidly convergent scalar twodimensional Green's function described in [1]. On the other hand, $M$ is the number of TM modes of the surrounding standard rectangular waveguide required by the modal expansion of the BI-RME method, and $k_{m}^{\prime}$ and $\psi_{\boldsymbol{m}}$ are, respectively, the cut-off wavenumber and normalised scalar potential of the $m$-th TM mode of such rectangular waveguide. The unknowns of the problem are the modal expansion coefficients $\left(a_{m}^{\prime}\right)$, the amplitudes of the longitudinal current density in the discrete points $\left(\mathbf{s}_{j}\right)$ of the contour $\left(b_{j}^{\prime}\right)$, and the cut-off wavenumbers of the perturbed waveguide $\left(k^{\prime}\right)$.

In this approach, a simple one point quadrature is chosen for $\omega_{j i}$ and $\gamma_{j}$. However, as can be seen in (2a), the Nyström method requires the evaluation of the kernel with coincident source and observation points. In such cases, the singular contribution of the kernel is approximated by its Taylor expansion, which gives place to a regular and a singular term. To compute the corresponding element $\omega_{i i}$, the regular term is numerically integrated, whereas the singular one is analytically solved. Further details for each kind of segment (i.e. straight, circular and elliptical) will be presented during the talk.

\section{B. TE Modes}

For solving the TE case, the original BI-RME formulation is modified (see (8a) and (8b) in [2]), since the transverse dyadic involving the double gradient of the Green's function $g$ is preserved. Proceeding in this way, the unknown transverse current density does not involve any derivative, thus easing the numerical solution. Therefore, the following set of algebraic equations is obtained

$$
\begin{aligned}
& \frac{1}{k^{2}} \sum_{\substack{j=1 \\
j \neq i}}^{N} \frac{\partial^{2} g\left(\mathbf{s}_{i}, \mathbf{s}_{j}\right)}{\partial l \partial l^{\prime}} \omega^{\prime}{ }_{i j} b_{j}-\sum_{\substack{j=1 \\
j \neq i}}^{N} \mathbf{t}\left(\mathbf{s}_{i}\right) \mathbf{G}_{s t}\left(\mathbf{s}_{i}, \mathbf{s}_{j}\right) \mathbf{t}\left(\mathbf{s}_{j}\right) v_{i j} b_{j} \\
& +\frac{1}{k^{2}} \omega^{\prime}{ }_{i i} b_{i}-v_{i i} b_{i}-\sum_{m=1}^{M} \frac{\mathbf{t}\left(\mathbf{s}_{i}\right) \mathbf{e}_{m}\left(\mathbf{s}_{i}\right)}{k_{m}^{2}} a_{m}=0 \quad(i=1, . ., N) \\
& \left(1-\frac{k^{2}}{k_{m}^{2}}\right) \cdot a_{m}=k^{2} \sum_{j=1}^{N} \frac{\mathbf{e}_{m}\left(\mathbf{s}_{j}\right) \mathbf{t}\left(\mathbf{s}_{j}\right)}{k_{m}^{2}} \cdot \gamma_{j}^{\prime} b_{j} \quad(m=1, \ldots, M)
\end{aligned}
$$

where $\mathbf{G}_{s t}$ is now the rapidly convergent solenoidal dyadic normal to the boundary described in [2], and $\mathbf{t}$ is the unitary tangent vector on the arbitrary contour. In this case, $M$ represents the number of TE modes of the surrounding standard rectangular waveguide, and $k_{m}$ and $\psi_{m}$ are, respectively, the cut-off wavenumber and normalised scalar potential of the $m$-th TE mode of the rectangular waveguide. In this problem, the unknowns are again the modal expansion coefficients $\left(a_{m}\right)$, the amplitudes of the transverse current density in the discrete points $\left(\mathbf{s}_{j}\right)$ of the contour $\left(b_{j}\right)$, and the cut-off wavenumbers of the perturbed waveguide $(k)$.

The quadrature weights $\omega_{j i}^{\prime}, v_{i i}, v_{j i}$ and $\gamma_{j}^{\prime}$ coefficients of the Nyström method are obtained in the same way as explained before for the TM case. However, as it can be seen in (3a), the computation of the $\omega^{\prime}{ }_{i i}$ coefficients involve a double partial derivative of $g$ with respect to the observation and source contour parameters $l$ and $l^{\prime}$ is required, which is not integrable even in the Cauchy principal value definition. Therefore, the treatment of this singularity is different with regard to the previous case. In this case, $\omega^{\prime}$ ii is computed via the traditional method of the subtraction of the singularity because of the hypersingularity of the double derivative of Green's function.

\section{RESULTS}

The new algorithm has been tested on different geometries in order to identify the advantages of the method compared to standard Galerkin BI-RME method [5]. All tests have been performed on a PC Pentium II @ $400 \mathrm{MHz}$. Obviously, the Nyström method with onepoint quadrature should need more discrete points than Galerkin's technique in order to obtain the same accuracy. Therefore, the starting point is to use the same number of points $N$ than the Galerkin case. The second step is to compute the relative error in the computation of the cut-off frequencies. The maximum relative error allowed is set to $0.5 \%$ when compared to Galerkin BIRME. If that bound is not satisfied, then the spatial grid is increased. As a consequence, the benchmark results depend on several factors, such as the geometry, the size of the perturbation contour and the desired number of valid modes.

The validity of this new method can also be proved by representing the axial electric field for the Transversal Magnetic (TM) modes and the transversal electric field for the Transversal Electric (TE) modes. Finally, in order to confirm the accuracy of the novel technique several coupling coefficients will be computed.

\section{A. Ridge waveguide}

The first geometry to be tested is a WR-75 standard waveguide perturbed by a central single ridge shown in Fig. 1. The results obtained are shown in Table I. Time savings are achieved with a small loss of accuracy and the same number of points $N$ as in Galerkin. In this comparison, 600 modes in the rectangular waveguide are used and 140 single ridge valid cut-off frequencies are obtained. The mean and maximum relative error of these 140 cut-off frequencies are also shown in Table I.

The electric field obtained in this example is shown in Fig. 2a) for the axial component in the $4^{\text {th }} \mathrm{TM}$ mode, while Fig. 2b) shows the transversal component for the $4^{\text {th }}$ TE mode. 


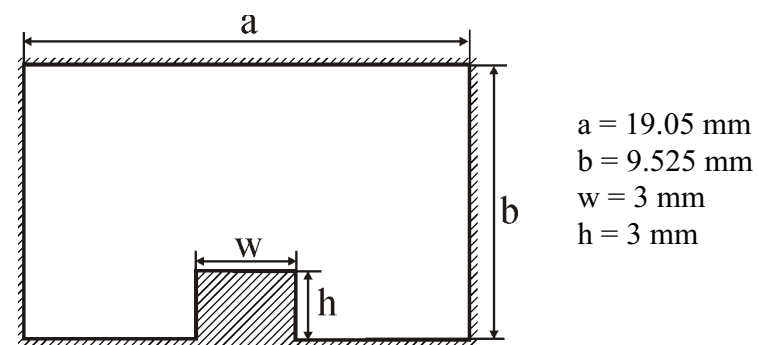

Fig. 1. Single ridge waveguide geometry.

TABLE I. Single Ridge WAVEGUIDE RESUlts

\begin{tabular}{|c|c|c|c|c|c|}
\hline & $\begin{array}{l}\text { N extra } \\
\text { points }\end{array}$ & $\begin{array}{c}\text { Time } \\
\text { reduction }\end{array}$ & Time & $\begin{array}{c}\text { Max. } \\
\text { Error }\end{array}$ & $\begin{array}{c}\text { Mean } \\
\text { error }\end{array}$ \\
\hline TM & $0 \%$ & $38.9 \%$ & $1.98 \mathrm{~s}$ & $0.05 \%$ & $0.02 \%$ \\
\hline TE & $0 \%$ & $32 \%$ & $6.12 \mathrm{~s}$ & $0.19 \%$ & $0.05 \%$ \\
\hline
\end{tabular}
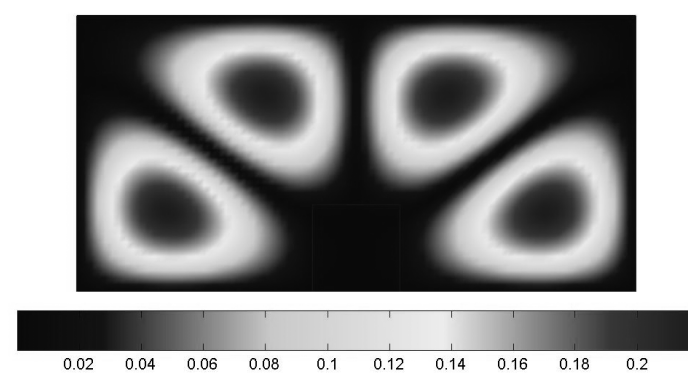

a)

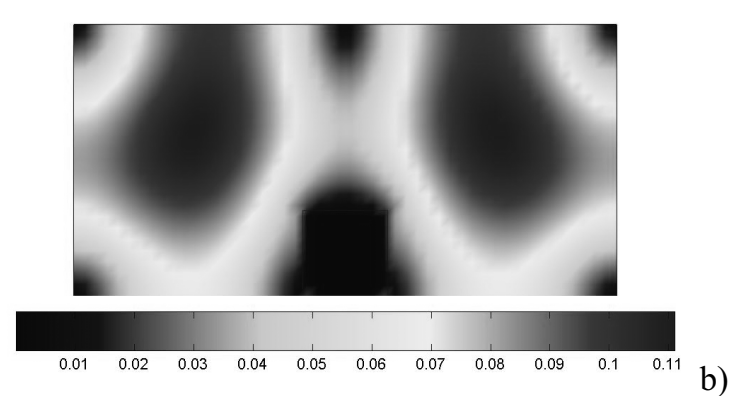

Fig. 2. Electric field representation for the ridge waveguide.

As it is expected, the computed fields are practically zero outside the region of interest as was expected.

For this case, the coupling integrals for the first 8 modes of the single ridge and 21 modes of a WR-75 waveguide have also been computed. A maximum absolute error of $4 \cdot 10^{-3}$ has been observed, thus confirming the accuracy of the novel technique. Table II shows some coefficients and their associate absolute error.

\section{B. Multiridge waveguide}

In this geometry, a rectangular waveguide is perturbed by six ridges symmetrically distributed (see Fig. 3). As can be seen in Table III, the computing time reduction for the TM case is very large. In the case of TE modes, the result is not so impressive because the grid had to be increased with extra $50 \%$ discrete points in order to keep the error bound. Despite of the spatial grid expansion required, the total computation effort is still reduced. In this case, the number of rectangular waveguide modes is 600 , and 110 valid modes are obtained.

\begin{tabular}{|c|c|c|c|c|c|}
\hline \multirow{2}{*}{$\begin{array}{c}\text { Modes } \\
\text { A }\end{array}$} & \multicolumn{5}{|c|}{ Modes B } \\
\hline & $\mathrm{TE}_{10}$ & $\mathrm{TE}_{20}$ & $\mathrm{TE}_{01}$ & $\mathrm{TE}_{11}$ & $\mathrm{TM}_{11}$ \\
\hline $\mathrm{TE}_{10}$ & $\begin{array}{l}-0.851 \\
1.7 \cdot 10^{-3}\end{array}$ & 0 & $\begin{array}{c}-0.213 \\
2.3 \cdot 10^{-3}\end{array}$ & 0 & -- \\
\hline $\mathrm{TE}_{01}$ & 0 & $\begin{array}{c}-0.7619 \\
2 \cdot 10^{-4}\end{array}$ & 0 & $\begin{array}{r}-0.593 \\
1 \cdot 10^{-3}\end{array}$ & -- \\
\hline $\mathrm{TE}_{20}$ & 0 & $\begin{array}{c}-0.602 \\
1.4 \cdot 10^{-3} \\
\end{array}$ & 0 & $\begin{array}{c}0.7649 \\
6 \cdot 10^{-4} \\
\end{array}$ & -- \\
\hline $\mathrm{TE}_{11}$ & $\begin{array}{c}-0.0269 \\
2.2 \cdot 10^{-4} \\
\end{array}$ & 0 & $\begin{array}{c}0.918 \\
1.2 \cdot 10^{-3} \\
\end{array}$ & 0 & -- \\
\hline $\mathrm{TM}_{11}$ & $\begin{array}{l}0.329 \\
4 \cdot 10^{-3}\end{array}$ & 0 & $\begin{array}{c}-0.186 \\
2 \cdot 10^{-3}\end{array}$ & 0 & $\begin{array}{c}- \\
0.768 \\
1 \cdot 10^{-3}\end{array}$ \\
\hline $\mathrm{TE}_{21}$ & 0 & $\begin{array}{c}0.0084 \\
3 \cdot 10^{-4}\end{array}$ & 0 & $\begin{array}{c}- \\
0.2134 \\
3 \cdot 10^{-4}\end{array}$ & -- \\
\hline $\mathrm{TM}_{21}$ & 0 & $\begin{array}{c}0.067 \\
2.1 \cdot 10^{-3}\end{array}$ & 0 & $\begin{array}{c}- \\
0.0108 \\
2 \cdot 10^{-4}\end{array}$ & 0 \\
\hline $\mathrm{TE}_{30}$ & $\begin{array}{l}0.0203 \\
1.7 \cdot 10^{-4}\end{array}$ & 0 & $\begin{array}{c}-0.129 \\
1.1 \cdot 10^{-3}\end{array}$ & 0 & -- \\
\hline
\end{tabular}

TABLE II. COUPLING COEFFICIENTS BETWEEN RECTANGULAR (A) AND RIDGE WAVEGUIDE MODES (B)

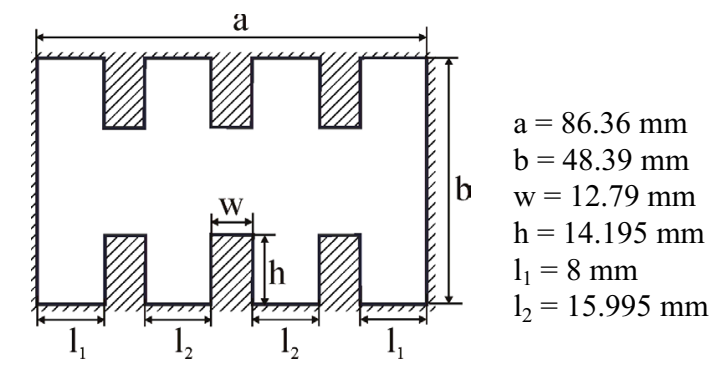

Fig. 3. Multiridge waveguide.

TABLE III. MULTI-RIDGE WAVEGUIDE RESULTS

\begin{tabular}{|c|c|c|c|c|c|}
\hline & $\begin{array}{l}\mathrm{N} \text { extra } \\
\text { points }\end{array}$ & $\begin{array}{c}\text { Time } \\
\text { reduction }\end{array}$ & Time & $\begin{array}{c}\text { Max. } \\
\text { Error }\end{array}$ & $\begin{array}{c}\text { Mean } \\
\text { error }\end{array}$ \\
\hline TM & $0 \%$ & $92.2 \%$ & $3.67 \mathrm{~s}$ & $0.34 \%$ & $0.18 \%$ \\
\hline TE & $50 \%$ & $65.8 \%$ & $48.47 \mathrm{~s}$ & $0.49 \%$ & $0.23 \%$ \\
\hline
\end{tabular}

\section{Elliptic waveguide}

An elliptic waveguide enclosed by an auxiliary WR-90 rectangular waveguide (see Fig. 4) has also been analysed. The number of WR-90 waveguide modes is 800 , and 29 valid modes are solved for the elliptic guide (see results in Table IV). The error bounds are similar to the multi-ridge case. The TE problem needs again a more dense spatial grid in order to satisfy the error limitations, thus decreasing the time reduction. 


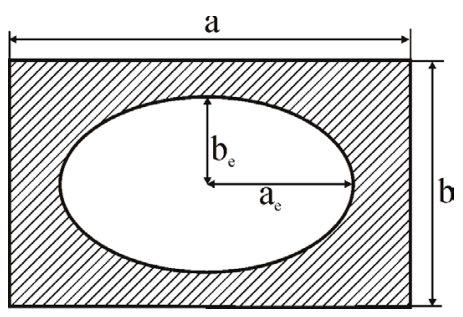

$\mathrm{a}=22.86 \mathrm{~mm}$

$\mathrm{b}=10.16 \mathrm{~mm}$

$\mathrm{a}_{\mathrm{e}}=5 \mathrm{~mm}$

$\mathrm{b}_{\mathrm{e}}=2.1794 \mathrm{~mm}$

Fig. 4. Elliptic waveguide geometry.

TABLE IV. ELLIPTIC WAVEGUIDE RESULTS

\begin{tabular}{|c|c|c|c|c|c|}
\hline & $\begin{array}{l}\text { N extra } \\
\text { points }\end{array}$ & $\begin{array}{c}\text { Time } \\
\text { reduction }\end{array}$ & Time & $\begin{array}{c}\text { Max. } \\
\text { error }\end{array}$ & $\begin{array}{c}\text { Mean } \\
\text { error }\end{array}$ \\
\hline TM & $0 \%$ & $59.7 \%$ & $6.41 \mathrm{~s}$ & $0.33 \%$ & $0.21 \%$ \\
\hline TE & $50 \%$ & $26.5 \%$ & $34.34 \mathrm{~s}$ & $0.48 \%$ & $0.26 \%$ \\
\hline
\end{tabular}

\section{Rounded Corner waveguide}

A WR-75 waveguide perturbed by four rounded corners (see Fig. 5) has been considered. The time reduction and the error bound is excellent for the TM case even without grid expansion, as shown in Table V. The TE mode computation gives a larger error but the spatial grid does not need to be modified with respect to the Galerkin case. The number of rectangular waveguide modes is 600 , and in this case 147 valid modes have been obtained for the perturbed waveguide.

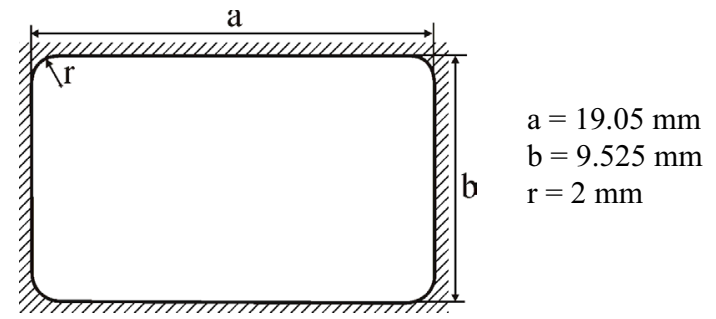

„Fig. 5. Rectangular waveguide with rounded corners.

TABLE V. RECTANGULAR WAVEGUIDE WITH ROUNDED CORNERS RESULTS

\begin{tabular}{|c|c|c|c|c|c|}
\hline & $\begin{array}{l}\text { N extra } \\
\text { points }\end{array}$ & $\begin{array}{c}\text { Time } \\
\text { reduction }\end{array}$ & Time & $\begin{array}{c}\text { Max. } \\
\text { error }\end{array}$ & $\begin{array}{c}\text { Mean } \\
\text { error }\end{array}$ \\
\hline TM & $0 \%$ & $41.2 \%$ & $1.8 \mathrm{~s}$ & $0.05 \%$ & $0.02 \%$ \\
\hline TE & $0 \%$ & $19.5 \%$ & $12.43 \mathrm{~s}$ & $0.32 \%$ & $0.09 \%$ \\
\hline
\end{tabular}

The electric field representation for the case of the rounded corner waveguide can be seen in Fig. 6, where the axial component for the fourth and the sixth TM modes are shown.

\section{CONCLUSIONS}

A new BI-RME algorithm based on the Nyström method has been successfully implemented and tested with different complex geometries. The main difficulty of this new approach comes from the treatment of the singularities of the integral equation kernels, which are different in nature for the TM and the TE modes. It has been shown that the new method offers some advantages when compared to the original MoM BI-RME method. The first one is the simplicity of the implementation. The second advantage is related to the reduction of the computation time. Therefore, this new method has been revealed as a good candidate for an efficient and less accurate simulator intended for a design tool. Furthermore, the Nyström method can be combined with the Wavelet transform in order to continue improving the computational efficiency.

\section{REFERENCES}

[1] G. Conciauro, M. Guglielmi and M. Sorrentino, Advanced modal Analysis - CAD Techniques for Waveguide Ciomponents and Filters, Chichester: John Wiley and Sons, Ltd. 2000.

[2] G. Conciauro, M. Bressan and C. Zuffada, "Waveguide modes via an integral equation leading to a linear matrix eigenvalue problem". IEEE Trans. Microwave Theory Tech, vol. MTT-32, no. 11, pp. 1495-1504, Nov. 1984.

[3] P. Arcioni, "Fast evaluation of modal coupling coefficients of waveguide step discontinuities". IEEE Microwave and Guided Wave Lett., vol. 6, no. 6, pp. 232-234, June 1996.

[4] J.W. Bandler, R. Biernacki, S.H. Chen, R. Hemmers and K. Madsen, "Electromagnetic optimization exploiting aggressive space mapping". IEEE Trans. Microwave Theory Tech, vol. MTT-43, no. 12, pp. 2874-2881, Dec. 1985.

[5] S. Cogollos, S. Marini, P. Soto, H. Esteban, J.V. Morro, V. Boria and B. Gimeno, "Modal Computation of Arbitrary Waveguides Composed of Linear, Circular and Elliptical Arcs", 2003 IEEE MTT-S Int. Microw. Symp., Philadelphia USA, June 2003. Accepted for publication.

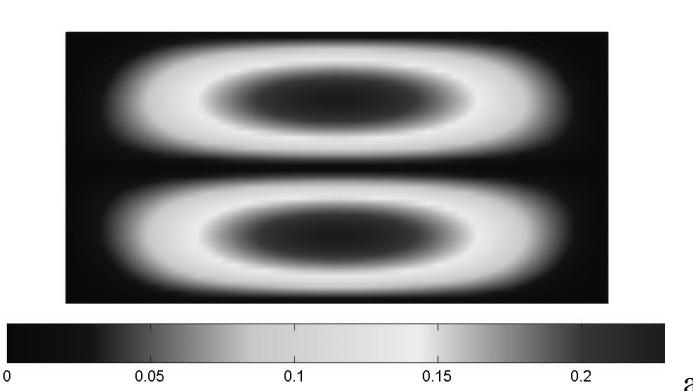

a)

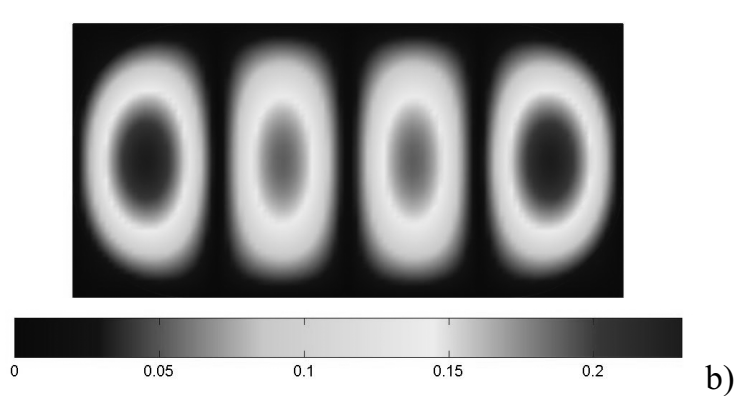

Fig. 6. Electric Field representation for the rounded corner waveguide for the $4^{\text {th }}$ mode in a) and the $6^{\text {th }}$ mode in b). 